\title{
Parkinson SEMG signal prediction and generation with Neural Networks
}

\author{
Rafael Anicet Zanini ${ }^{1}$ and Esther Luna Colombini ${ }^{1}$ \\ ${ }^{1}$ Laboratory of Robotics and Cognitive Science (LaRoCS), \\ Universidade Estadual de Campinas (UNICAMP) \\ Campinas - SP, 13083-852, Brazil; \\ E-mail: rzanini@gmail.com, esther@ic.unicamp.br
}

\begin{abstract}
Resumo. A Doença de Parkinson (DP) é uma doença neurodegenerativa caracterizada por sintomas como tremores de repouso e de ação, que causam graves prejuízos à vida do paciente. Recentemente, diversas dispositivos assistivos têm sido propostos para minimizar o impacto da doença na vida dos pacientes. No entanto, a maioria desses depende de dados da eletromiografia de superfície (sEMG) do paciente, que são escassos. Neste trabalho, propomos os primeiros métodos, baseados em Redes Neurais, para prever e gerar sinais de sEMG de pacientes com Parkinson (PP). Ainda, aprendemos o estilo do tremor no sinal de sEMG destes pacientes, transferindo o mesmo para criar novas amostras a partir de dados de pacientes saudáveis. Esta dissertação contribui para a área i) comparando diferentes modelos para predizer sinais $S E M G$ de pacientes com Parkinson para antecipar padrões de tremor em repouso; ii) propondo a primeira abordagem baseada em Redes Adversariais Generativas Convolucionais Profundas (DCGANs) para gerar sinais de sEMG da doença de Parkinson; iii) aplicando a transferência de estilo (ST) para aumentar o conjunto de sinais sEMG de PPs a partir de dados disponíveis publicamente de indivíduos nãoPP; iv) propondo uma métrica para avaliar a caracterização do sinal de sEMG da doença de Parkinson. Os novos dados criados por nossos métodos podem validar abordagens de tratamento em diferentes cenários de movimento, contribuindo para o desenvolvimento de novas técnicas de supressão de tremor em pacientes.
\end{abstract}

\section{Introdução}

Como uma das doenças neurodegenerativas mais comuns que afetam aproximadamente 10 milhões de pessoas em todo o mundo [Organization. 2006], a Doença de Parkinson (DP) tem sido estudada e investigada de diferentes perspectivas para minimizar os sintomas da doença e prejuízos aos pacientes, com muitos estudos em torno de tremores de repouso e ação. A eletromiografia de superfície (sEMG) se destaca como uma das formas mais comuns de medir a resposta muscular à estimulação voluntária ou involuntária, sendo amplamente utilizada como sinal de feedback de entrada primária para dispositivos de estimulação artificial [Bó 2010] e empregada recentemente para várias aplicações de interface homem-máquina. Normalmente, essas aplicações exigem conjuntos de dados para treinar modelos de Aprendizado de Máquina (AM).

No entanto, adquirir tais conjuntos de dados de pacientes é uma tarefa complicada e às vezes dolorosa, e uma ampla gama de movimentos geralmente não é possível devido à limitação e deficiência de movimento do paciente. Portanto, coletar, processar 
e empregar sinais de sEMG de pacientes com Parkinson em modelos de AM pode ser desafiador devido à escassez e à falta de variação do conjunto de dados. Nesse cenário, simulações de sinais biológicos podem ser empregadas. No entanto, a geração de modelos realistas requer um conhecimento profundo dos padrões e morfologia dos sinais simulados [Petersen and Rostalski 2019]. Além disso, uma vez que os padrões de tremor da DP diferem em intensidade e forma para cada paciente, é bastante desafiador criar um modelo matemático genérico [Guerrero and Macías-Díaz 2019] que possa efetivamente produzir um sinal artificial semelhante aos reais. Além disso, tal abordagem não pode se adaptar a diferentes protocolos de movimento ou se ajustar ao padrão de tremor específico de um indivíduo, cujos parâmetros não são conhecidos a priori e apresentam irregularidades típicas nas frequências e forma ao longo do tempo. Para superar essas restrições, o aumento de dados é uma abordagem alternativa para estender os conjuntos de dados existentes. Neste trabalho, empregamos modelos de redes neurais (NN) para prever, gerar e transferir o estilo de sinais de tremor sEMG PP-específicos do paciente.

\section{Contribuições}

Esta dissertação propõe uma Abordagem baseada em redes neurais para prever os picos de tremores futuros de SEMG de pacientes com Parkinson. Para este fim, projetamos e avaliamos várias arquiteturas para prever sinais brutos e de envelope de sEMG com diferentes janelas de predição. A abordagem resultante foi publicada na IEEE International Conference on Systems, Man and Cybernetics 2018 [Zanini et al. 2019].

Também projetamos o primeiro método para gerar sinais de sEMG da doença de Parkinson. Ao empregar Redes Profundas Convolucionais Adversariais Generativas (DCGANs) com pipelines discriminadores específico ao domínio, simulamos com sucesso o comportamento do tremor sEMG, não apenas imitando padrões de tremor genéricos, mas características específicas do paciente e do protocolo empregado. Com esta abordagem, poderíamos gerar novos sinais sEMG capazes de lidar com problemas típicos em abordagens generativas, como mode collapse. Também abordamos como diferentes características de entrada, como FFT e Wavelet, afetam a qualidade do sinal gerado.

Finalmente, propomos uma abordagem de transferência de estilo (ST) para aumentar os sinais sEMG de Parkinson combinando dois bancos de dados de sEMG distintos. Para isso, usamos o discriminador treinado anteriormente como um extrator de características dos componentes do sinal de Parkinson. Este discriminador é usado em uma arquitetura de ST para combinar os componentes de sinal de PP aprendido aos dados do conjunto de dados NinaPro (sinais não-PP), criando um novo conjunto de dados. Para avaliar a qualidade do sinal final gerado, propomos usar como métricas para avaliação do sinal gerado a distância DTW, FFT MSE e correlação cruzada do envelope sEMG. Os resultados desses métodos foram publicados no Sensors Journal no trabalho "Parkinson's Disease EMG Data Augmentation and Simulation with DCGANs and Style Transfer''[Zanini and Colombini 2020].

\section{Predição de Sinal de sEMG}

A predição de sinais sEMG da doença de Parkinson com base nas leituras do paciente como entrada é um problema típico de predição de sequência de série temporal. Uma vez 


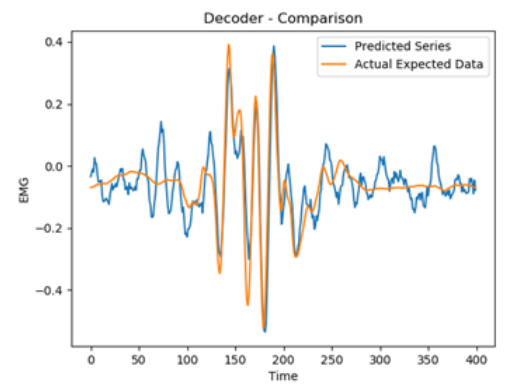

(a)

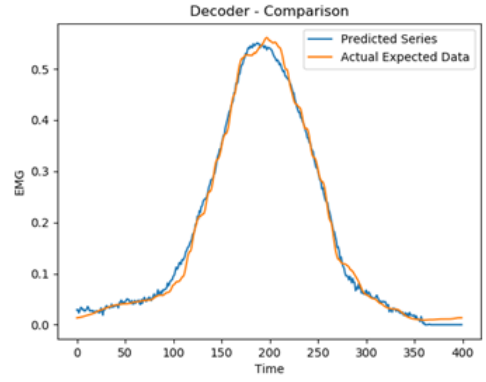

(b)

Figura 1. (a) Sinal previsto com rede MLP para o sinal bruto. (b) Sinal previsto com rede MLP decoder para o envelope sEMG.

que a frequência típica do tremor varia entre 4-6 Hz, cada estímulo de tremor acontece em uma janela de 0,2 segundos. Considerando uma taxa de amostragem de $2 \mathrm{kHz}$, precisamos cobrir pelo menos 400 pontos no futuro para prever quando o próximo estímulo de tremor acontecerá.

Para fazer isso, geramos 400 pontos no futuro com base nos últimos 4.000 pontos, usando duas abordagens: primeiro, usamos os dados SEMG brutos como entrada e avaliamos diferentes modelos baseados em MLP, LSTM e Autoencoders como um modelo de previsão. Essa abordagem pode nos dar uma boa estimativa da forma e do comportamento do sinal sEMG, mas não oferece uma boa estimativa da amplitude completa do tremor. Para prever melhor os picos e amplitudes do tremor, empregamos uma segunda abordagem, usando uma representação de envelopes de sEMG; usando os mesmos modelos para prever a forma e a amplitude do próximo envelope de sEMG. Resultados. Os resultados experimentais (Figura 1) mostraram que as redes baseadas em MLP e LSTM podem prever com sucesso o comportamento do tremor sEMG, tanto para o envelope quanto para o sinal bruto. Usando a abordagem do autoencoder, foi possível compactar informações de sEMG em um espaço de dimensão inferior. Também comparamos diferentes topologias MLP e LSTM, avaliando a influência dos hiperparâmetros nos modelos e como a função de erro afeta a qualidade da previsão, propondo uma nova métrica de erro para avaliar e treinar modelos de previsão de sEMG.

\section{Geração de Sinal de sEMG}

Em nosso trabalho, foram propostos dois métodos para aumento de dados de sEMG. No primeiro, baseado em DCGANs, treinamos um gerador capaz de simular o padrão de tremor sEMG de cada paciente e seu discriminador correlacionado. No segundo, com base na transferência de estilo neural e no discriminador treinado do método anterior, aplicamos o estilo de um paciente com DP em um conjunto de sinais sEMG de paciente saudável, simulando o comportamento de tremor esperado em um conjunto diferente de movimentos. Também empregamos as mesmas entradas para treinar uma rede de Transformers based on Fast Neural Style Transfer. A Figura 2 apresenta um diagrama simplificado dos métodos propostos.

\subsection{Geração de sinal de sEMG com DCGANs}

Apesar do sucesso atual e dos resultados na geração de imagens, as DCGANs têm sido menos explorados em séries temporais e aplicações biológicas. Neste trabalho, adapta- 


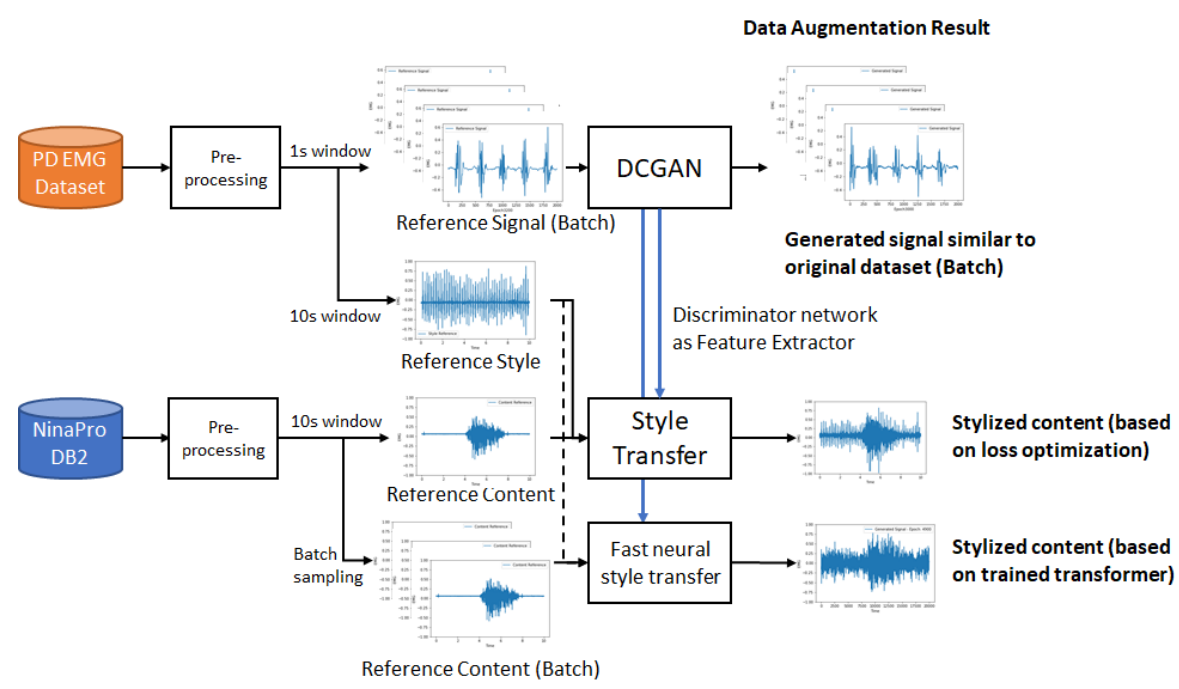

Figura 2. Fluxo proposto para os experimentos de geração de sinal de sEMG.

mos seu uso para o contexto 1D de múltiplas variáveis cujos padrões variam no tempo. Normalmente, ao criar GANs, o gerador é de interesse primário e o discriminador é uma função de erro adaptativa descartada uma vez que o gerador foi treinado. No entanto, como apresentamos neste trabalho, o discriminador treinado também pode ser usado como um extrator de características que pode ser aplicado em combinação com outras técnicas, como transferência de estilo.

Modelo do Gerador. Nosso melhor modelo gerador consiste em uma rede de convolução profunda - adaptada para convoluções 1D - que obtém amostras de 400 pontos $(0,2 \mathrm{~s})$ da amostra original e gera um novo conjunto de dados com 2.000 pontos (1 s). O modelo inclui uma camada densa e média móvel no final do pipeline do gerador para suavizar o sinal gerado em comparação com o sinal de entrada sEMG filtrado. A arquitetura final resulta de uma avaliação criteriosa sobre diferentes parâmetros como o número de filtros, camadas, funções de ativação e outras configurações.

Modelo do Discriminador. Nosso melhor modelo discriminador consiste em uma rede de convolução profunda que emprega um lote de 100 amostras distribuídas aleatoriamente com 2.000 pontos sequenciais e tenta distinguir se eles vêm do conjunto de dados de treinamento ou do gerador. Combinamos pipelines convolucionais profundos paralelos para essa tarefa, onde cada um gera características estendidas baseadas nos vetores de entrada. O pipeline combina quatro pilhas convolucionais com uma camada densa final para classificação entre amostras reais e falsas, sendo essas i) Filtros Convolucionais no Sinal Bruto; ii) Filtros convolucionais da FFT; iii) Filtros convolucionais do sinal de envelope da sEMG e iv) Filtros convolucionais da expansão wavelet.

\subsection{Geração de sEMG a partir da transferência de estilo}

Neste trabalho, empregamos duas abordagens de transferência de estilo [Gatys et al. 2015] para combinar o sinal característico da doença de Parkinson com sinais de sEMG de pacientes saudáveis. Na primeira, modificamos o algoritmo introduzido em [Gatys et al. 2015] para trabalhar com dados de série temporal 1D, ajustando as funções de perda de conteúdo e perda de estilo propostas para nosso 
domínio. Também substituímos a VGG16 original usada em [Gatys et al. 2015] pela rede discriminadora treinada usada para a arquitetura DCGAN. Empregamos as quatro pilhas convolucionais do discriminador principal (sinal bruto, FFT, FFT sobre envelopes e expansão wavelet) como as camadas de características para a função de erro do estilo, calculando a gram matrix para os filtros convolucionais do sinal de estilo e o sinal gerado. Para a segunda abordagem, usamos o conceito de fast neural style transfer [Johnson et al. 2016] para treinar uma rede de Transformers. Esta rede recebe um sinal de sEMG de um indivíduo saudável como entrada - realizando algumas ações (como flexão/extensão do punho, agarrar, apontar o dedo indicador e outros) - e aplica uma transformação com base no sinal de sEMG de paciente com DP para simular a aparência do sinal se realizado por um paciente com DP. A rede é treinada com base em um conjunto de exemplos de conteúdo (banco de dados NinaPro de indivíduos saudáveis) e o estilo (sinais de sEMG de nosso conjunto privado de dados de pacientes com DP). Para calcular o erro entre conteúdo, estilo e sinais transferidos, usamos o discriminador pré-treinado da DCGAN como um extrator de características - permitindo a rede aprender os padrões individuais de cada paciente, de acordo com o discriminador e gerador treinados. Comparamos essa abordagem com o modelo baseado em ST.

\subsection{Métricas propostas}

Avaliamos a qualidade dos sinais gerados propondo três métricas para o nosso domínio. O erro quadrado médio (MSE) entre as magnitudes dos sinais FFT foi usado para medir a semelhança entre os dados gerados e os dados reais, avaliando a semelhança entre o sinal gerado e os sinais de estilo e componentes na etapa de transferência de estilo. O Dynamic Time Warping (DTW) é um método que mede a similaridade entre duas sequências temporais pela comparação das funções de custo local de ambas as sequências. Delaney et al. (2019) mostraram que a DTW pode avaliar com sucesso a qualidade dos dados gerados, pois esta é mais robusta à instabilidade do treinamento e sensibilidade à amplitude relativa entre os dados reais e sintéticos. A função de correlação cruzada é uma medida de similaridade de duas séries em função do deslocamento de uma em relação à outra, sendo bastante usada na neurofisiologia [Semmlow and Griffel 2014]. Uma vez que a forma e amplitude do tremor em sEMG podem variar significativamente entre os sinais de referência e gerados, identificamos que a correlação cruzada simples em sinais brutos não capturaria a similaridade entre eles. Portanto, calculamos a correlação cruzada normalizada entre os envelopes de EMGs (com uma média móvel de 100 pontos em valores absolutos) para verificar se os sinais gerados capturaram corretamente os picos de tremor.

\subsection{Resultados}

Nos experimentos de geração de sinal, chegamos a descobertas significativas relacionadas a bio-sinais 1D complexos, como sinais de sEMG. Em primeiro lugar, enfatizamos que, embora empregando uma abordagem baseada em Deep Learning, onde características podem ser aprendidas diretamente do sinal, foi essencial adicionar diferentes pipelines convolucionais que se concentram em características específicas do sinal para produzir saídas complexas. Em nosso caso, adicionar a análise FFT e a decomposição de Wavelet ao modelo discriminador, bem como o uso de convoluções 1D foi essencial para gerar melhores resultados. Também verificamos a importância de métricas que podem avaliar efetivamente o desempenho do gerador em relação aos sinais de referência. 
FFT e DWT foram as melhores métricas de avaliação para nosso domínio. Também foi relevante descobrir que poderíamos empregar nosso discriminador como um extrator de características significativo para a técnica de transferência de estilo. Finalmente, encontrar pesos apropriados para os sinais de conteúdo e estilo é uma tarefa desafiadora. Em nosso caso, seu impacto poderia ser avaliado mais detalhadamente se sinais reais de pacientes com a doença de Parkinson realizando os mesmos movimentos de indivíduos saudáveis estivessem disponíveis. Todos os resultados podem ser consultados no trabalho completo [Zanini 2020], disponível no acervo digital da UNICAMP (http: //repositorio.unicamp.br/jspui/handle/REPOSIP/346211).

\section{Principais Avanços no Estado da Arte}

Na predição e geração do sinal sEMG de pacientes com doença de Parkinson, criamos os primeiros métodos para tais tarefas. Também empregamos a primeira abordagem baseada em transferência de estilo para combinar um estilo de sinal típico de uma doença neurodegenerativa com o sinal de um paciente saudável. É importante ressaltar que, embora validemos nossos achados no contexto da Doença de Parkinson, os modelos propostos podem ser empregados em outros cenários baseados em sEMG. Também levantamos a hipótese de que os sinais de EEG e ECG poderiam ser usados em vez de sEMG. O código está disponível em nosso repositório público www. gi thub.com \larocs

\section{Referências}

Bó, A. P. L. (2010). Compensation active de tremblements pathologiques des membres supérieurs via la stimulation électrique fonctionnelle. $\mathrm{PhD}$ thesis, Université Montpellier II.

Gatys, L. A., Ecker, A. S., and Bethge, M. (2015). A neural algorithm of artistic style. ArXiv, abs/1508.06576.

Guerrero, A. and Macías-Díaz, J. E. (2019). A package for the computational analysis of complex biophysical signals. International Journal of Modern Physics $C$, 30(01):1950005.

Johnson, J., Alahi, A., and Fei-Fei, L. (2016). Perceptual losses for real-time style transfer and super-resolution. In $E C C V$.

Organization., W. H. (2006). Neurological disorders: public health challenges.

Petersen, E. and Rostalski, P. (2019). A comprehensive mathematical model of motor unit pool organization, surface electromyography, and force generation. Frontiers in Physiology, 10:176.

Semmlow, J. L. and Griffel, B. (2014). Biosignal and Medical Image Processing. CRC Press.

Zanini, R. A. (2020). Previsão e geração de sinais emg de parkison com redes neurais. Master's thesis, UNICAMP.

Zanini, R. A. and Colombini, E. L. (2020). Parkinson's disease emg data augmentation and simulation with degans and style transfer. Sensors, 20(9).

Zanini, R. A., Colombini, E. L., and de Castro, M. C. F. (2019). Parkinson's disease emg signal prediction using neural networks. In 2019 IEEE International Conference on Systems, Man and Cybernetics (SMC), pages 2446-2453. 